\title{
Ersetzt die Neue Ökonomische Geographie Außenwirtschaftstheorie und Raumwirtschaftstheorie?
}

\author{
von \\ Klaus Schöler \\ Wirtschafts- und Sozialwissenschaftliche Fakultät \\ der Universität Potsdam
}

Diskussionsbeitrag Nr. 100/2010

\section{Inhalt}

1. Einführung

2. Außenwirtschaftstheorie vs. Raumwirtschaftstheorie

3. Was erklärt die Neue Ökonomische Geographie?

4. Fazit

Universität Potsdam, Wirtschafts- und Sozialwissenschaftliche Fakultät, Lehrstuhl für Volkswirtschaftslehre, insbesondere Wirtschaftstheorie, August-Bebel-Straße 89, 14482 Potsdam 


\section{Einführung}

Zunächst einige kurze wissenschaftstheoretische Überlegungen: Zur Beurteilung von Theorien gelten in den Naturwissenschaften - aber auch in den Sozialwissenschaften einfache, leicht nachzuvollziehende wissenschaftstheoretische Prinzipien. Jene Theorie, die einen größeren Ausschnitt der Realität zu erklären vermag als eine konkurrierende Theorie, ist dieser überlegen und also vorzuziehen. Bei gleichem Erklärungsgehalt von Theorien - was allerdings selten vorkommen dürfte - ist jene die überlegenere, die einfacher ist, wobei einfacher bedeutet, daß sie mit weniger Annahmen, mit weniger Randbedingungen auskommt. Nun ist in der wissenschaftlichen Realität der beschriebene Prozeß kaum zu beobachten; rationale Prinzipien und tatsächliche Theorienabfolge und -ablösung stehen sich als Ideal und Wirklichkeit gegenüber. Dafür kann es verschiedene Gründe geben. Zum einen können die konkurrierenden Theorien aus unterschiedlichen Denkschulen stammen; eine Entscheidung zu Gunsten des einen Ansatzes und gegen ein anderes theoretisches Konzept ist mit dem wissenschaftlichen Schicksal der jeweiligen Forscher verbunden (vgl. Kuhn [1981]). Zum anderen können aber auch konkurrierende Theorien sich zunächst unterschiedlichen Phänomenen zuwenden, die in dieser Form dann im Zeitablauf empirisch unbedeutender werden. Im Gegenzug weiten sich die Erklärungshorizonte beider Theorien aus, und es kommt zur Schnittmenge der Erklärungsobjekte. Eine Konkurrenzsituation entsteht, die vielfach nicht einmal erkannt wird.

Der zweite der beschriebenen Gründe trifft in dieser Konstellation sehr genau auf das Verhältnis von Außenwirtschaftstheorie (auch: Internationaler Handel) und Raumwirtschaftstheorie (auch: Regionalökonomik) zu. Unter Außenwirtschaftstheorie soll hier einschränkend der reale Teil dieser Disziplin verstanden werden; von den monetären Theorien der Wechselkurse und Zahlungsbilanzen soll abgesehen werden, da sie aus wissenschaftssystematischen Gründen zur Geldtheorie gehören, und daher auch keine Entsprechung in der Raumwirtschaftstheorie haben. Zunächst fällt der gemeinsame Nenner auf: In beiden Ansätzen werden Wirtschaftsaktivitäten Regionen zugeordnet und die Interaktionen zwischen den Regionen analysiert. Es ist lohnenswert, einen genauen Blick auf die Grundelemente beider Theorien zu werfen und die gemeinsamen Eigenschaften herauszuarbeiten (Absatz 2). Nach diesem Vergleich wird der Frage nachgegangen, ob die Neue Ökonomische Geographie (kurz: NÖG) Eigenschaften aufweist, die die beiden "alten" Theorien ersetzen und somit überflüssig machen (Absatz 3). Ein Fazit - die Beurteilung der Theorien auf der Metaebene - schließt sich an (Absatz 4). 


\section{Außenwirtschaftstheorie vs. Raumwirtschaftstheorie}

Beginnen wir mit der Außenwirtschaftstheorie. Die Eingangsfrage lautet: Warum benötigt man eine Theorie des internationalen Handels? Die einzig mögliche Antwort lautet: weil Nationen, im eigentlichen Sinne staatliche Autoritäten, sich in den grenzüberschreitenden Handel einmischen (von den Wechselkursen wollen wir, wie gesagt, absehen) und diesen Güter- und Dienstleistungsaustausch im ökonomischen Sinn beeinflussen. Staatliche Handelshemmnisse und Handelsförderung dienen traditionell dazu, die eigene Wirtschaft vor Importen zu schützen und die Exporte zu fördern. Der Instrumentenkasten enthält bekanntlich Importzölle, Importkontingente, technische Anforderungen an Importgüter usw. auf der einen Seite und Exportsubventionen, Exportzölle und nichtmonetäre Exporthilfen auf der anderen Seite. Im Rahmen der modernen internationalen Handelsbeziehungen werden Zölle zunehmend abgebaut, es entstehen Freihandelszonen und Staatengemeinschaften (z. B. die EU). Wird damit die Außenwirtschaftstheorie um ihre Existenz gebracht? Keineswegs, denn wichtige Erklärungsmuster für den intersektoralen, aber auch intrasektoralen Handel bleiben bestehen. Zu nennen sind die unterschiedlichen Produktivitäten zwischen Ländern (oder einem Land und dem Rest der Welt) - das Ricardo-Theorem mit absoluten und komparativen Kostendifferenzen -, die unterschiedlichen Faktorausstattungen in verschiedenen Ländern - das Heckscher-Ohlin-Theorem - und die voneinander abweichenden Nachfrageintensitäten in den Ländern. Ersetzt man den Begriff "Länder" durch "Regionen", so ändert sich nichts am Ergebnis und an der Erklärungskraft der Theoreme. Entfällt der Staat mit seinen handelspolitischen Aktivitäten zur Kennzeichnung und Abgrenzung der nationalen Gebietseinheiten, so treten die genannten Unterschiede Produktivität, Faktorausstattung und Nachfrage an seine Stelle, die auf Gebietseinheiten jeglicher Größe bezogen werden können (Ohlin [1933]). Um Beispiele zu nennen: zwischen der EU und den USA, zwischen Deutschland und Portugal, zwischen Bayern und Brandenburg, zwischen dem Ruhrgebiet und dem Rhein-Main-Gebiet gibt es Produktivitätsunterschiede, die entsprechende Handelsströme erzeugen können. Gleiches gilt für die anderen Handelsargumente. Ist also die um den Staat entkleidete Außenwirtschaftstheorie die bessere Raumwirtschaftstheorie, die bekanntlich mit totalanalytischen Modellen arbeitet, die umfassender sind als die typischen partialanalytischen Ansätze der traditionellen Raumwirtschaftstheorie?

Es gibt einen entscheidenden Unterschied zwischen der traditionellen Raumwirtschaftstheorie und der Außenhandelstheorie; die Theorie des internationalen Handels verzichtet auf die Berücksichtigung der Transportkosten und überhaupt auf die Modellierung aller 
Transaktionskosten, die bei dem internationalen Güteraustausch entstehen. Es soll nicht abgestritten werden, daß es einige wenige Versuche in der Vergangenheit gab, diesen Mangel abzustellen (vgl. Steininer [2001]), hier soll von der generellen Linie gesprochen werden. Die Unterscheidung zwischen den Transportkosten und dem weiter gefaßten Begriff der Transaktionskosten soll kurz diskutiert werden. Um - sagen wir - eine Werkzeugmaschine nach Indien zu exportieren, ist es notwendig, wechselseitige Geschäftsbesuche durchzuführen, Verträge auszuarbeiten, Genehmigungen einzuholen, Dokumente zu übersetzen, Akkreditive zu eröffnen und schließlich fallen die reinen Land- und Seefrachttransportkosten an, nur diese sind im strengen Sinne entfernungsabhängig; alle anderen Transaktionskosten hängen von kulturellen Unterschieden der Länder, von Wirtschaftssystemen und staatlicher Einflußnahme u.d.m. ab. Will man einen ökonomischen Raum aufspannen, so sind die geographischen Entfernungen zwischen den Handelpartnern und ihren Heimatländern entscheidend. Genau diese Überlegungen finden wir in der traditionellen Außenhandelstheorie nicht, wofür zwei Gründe maßgeblich sein mögen. Zum einen gelten die oben genannten Gründe für Handel - Produktivitätsunterschiede, Faktorausstattungsunterschiede und Nachfrageunterschiede - bis zu jener Transportkostenhöhe (oder Transaktionskostenhöhe), bei der diese die handelserzeugenden Unterschiede eingeebnet haben. Alle Aussagen der reinen Außenwirtschaftstheorie stehen also unter dem stillschweigenden Vorbehalt, daß die genanten Kosten den Handel, ähnlich wie Zölle, nicht unvorteilhaft machen. Zum anderen reduziert die Außenhandeltheorie in ihren theoretischen Überlegungen - in sinnvoller Weise, will ich ausdrücklich hinzufügen - die Zahl der Industrien je Land auf zwei. Bei nicht vollständiger nationalen Produktionsspezialisierung stellt die x-Industrie im Inland das Exportgut und die y-Industrie das Importgut her; im Ausland ist es genau umgekehrt. Die Schwierigkeit besteht nun darin, einen dritten Sektor in die Modelle einzubeziehen, den Transportsektor, dessen Ressourcenverbrauch an die Import- und Exportmengen gebunden ist. Es gibt somit eine Rückkopplung, denn die Produktionsfaktoren, die für den Transport eingesetzt werden, stehen nicht mehr für die Produktion der x- und y-Güter zur Verfügung. Schließlich muß man von einem vertrauten Theorem Abschied nehmen; nach Aufnahme des Welthandels, ausgehend von dem gedachten Zustand der Autarkie, entsteht nicht ein gemeinsamer relativer Güterpreis im In- und Ausland, gleichsam als Welthandelspreisverhältnis, sondern relative Preise, die sich durch die Transportkosten unterscheiden. Die Analogie zur Zolltheorie liegt hier offen auf der Hand. Auch die Verwendung des Eisbergtheorems der Transportkosten von Samuelson ändert nicht grundlegend das Problem; lediglich die Formulierung des Transportsektors entfällt. 
Die Berücksichtigung der ökonomisch relevanten Transportkosten stellt das konstituierende Prinzip der Raumwirtschaftstheorie dar; dem aufgespannten ökonomischen Raum liegt ein geographischer Raum zu Grunde. Das bedeutet, einfacher gesagt, die geographischen Entfernungen zwischen den Wirtschaftsakteuren werden mit konstanten oder variablen Transportkostensätzen bewertet. Wir finden dieses Prinzip bei von Thünens "Isoliertem Staat" [1930], der die Verteilung der landwirtschaftlichen Standorte in Bezug auf ein Verbrauchszentrum bestimmt; wir finden es bei Launhardt [1885], der mit Hilfe der Transportkosten die Marktgebiete voneinander abgrenzt; wir finden es bei Weber [1909], für den die Transportkosten der Inputgüter bei gegebenen Faktorpreisen den industriellen Standort bestimmen, und wir finden es bei Christaller [1933] und Lösch [1944], deren hierarchische Standortstrukturen ohne die so verstandenen Transportkosten nicht möglich wären. In allen weiteren Theoriefeldern, die auf diesen grundlegenden Ansätzen aufbauen, die Stadtmodelle von Alonso [1964], die Beiträge zur optimalen Stadtgröße und die Überlegungen zur Preisbildung im Raum (vgl. Schöler [2005]) werden ebenfalls die beschriebenen Transportkosten als zentrales Element verwendet.

Vor nunmehr über siebzig Jahren hat August Lösch [1938, 1939] auf eine wichtige Verbindung von Raumwirtschaftstheorie und Außenwirtschaftstheorie hingewiesen. Lösch, der übrigens die auf den komparativen Kosten beruhende Handelstheorie Ricardos ablehnte, argumentierte wie folgt: Für ein Land kommen Exporte und Importe dadurch zustande, daß die überlappungsfreien Marktgebiete der Anbieter eines Gutes "zufällig" durch die Staatsgrenzen durchschnitten werden; jener Teil des Absatzgebietes eines inländischen Unternehmens, der ins Ausland fällt, generiert Exporte, jener Teil des Marktgebietes einer ausländischen Firma, der ins Inland fällt, erzeugt Importe. Werden Zölle eingeführt oder Exportsubventionen, so weist die räumliche Preislinie an der Staatsgrenze eine Sprungstelle auf. Die wohlfahrtsoptimale Ausprägung der handelspolitischen Instrumente läßt sich ermitteln; der traditionellen Formulierung des Optimalzolls kann nunmehr eine räumlich fundierte Fassung des Optimalzolls an die Seite gestellt werden, dessen Höhe von den Entfernungen der inländischen und ausländischen Produktionsstätten von der Staatsgrenze abhängt (Schöler [2005]). Damit wird deutlich: Transportkosten und Zölle haben für die heimische Wirtschaft eine schützende Wirkung und sind in der Lage, sich gegenseitig zu substituieren.

Ein ungelöstes Problem der Raumwirtschaftstheorie bestand bis in die neunziger Jahre des vorigen Jahrhunderts in der Erklärung des Entstehens von Agglomerationen. Wenn man von dem theoretisch trivialen Fall absieht, daß natürliche Gegebenheiten als exoge- 
ne Ursache für wirtschaftliche Zusammenballungen in Frage kommen, so muß man zugestehen, daß die endogenen Erklärungen auf schwachen eklektischen Füßen standen. Die theorieexogenen Erklärungen sind einfach nachzuvollziehen; wo Bodenschätze sich finden lassen, siedelt sich die Bergbauindustrie an; wo Flüsse vorhanden sind, bietet sich ihre Wasserkraft zur Energieerzeugung und zu Transportzwecken an, usw. Die endogenen Erklärungsversuche betonen die Existenz von positiven externen Effekten, die sich in vielfältigen Formen auswirken, wobei diese Effekte zwar in plausibler Form begründet, nicht aber aus Theorien entwickelt wurden. In der von-Thünen-Welt wird die Existenz eines Zentrums vorausgesetzt, auch in den nachfolgenden Modellen der Stadtökonomik (Alonso [1964]), wobei allenfalls die Größe der Stadt bestimmt werden kann. Das Christaller-Modell der Städtehierarchien ist der Agglomerationsbildung schließlich völlig unzugänglich. Obwohl Christaller den einzelnen Stadttypen idealtypische Einwohnerzahlen zuordnet, ist das Kriterium der Einwohnerzahl nicht konstituierend für die Stadtgröße, sondern allein die Reichweite der Güter, die nicht in einer Stadt mit geringerer Zentralität gehandelt werden.

\section{Was erklärt die Neue Ökonomische Geographie?}

Fragen wir nun, ob die Neue Geographie einen Beitrag zur Lösung der Probleme leisten kann, die wir sowohl in der Außenhandelstheorie - fehlende Transportkosten - als auch in der Raumwirtschaftstheorie - fehlende Erklärung der Agglomerationen - erkannt haben. Die Neue Ökonomische Geographie bietet zum einen eine modellendogene Erklärung für das Phänomen der Agglomeration und gleichzeitig für das entleerte Hinterland an, die aus einem totalanalytischen Modell mit wenigstens zwei Regionen abgeleitet wird. Zum anderen ist aus dem Modell heraus nicht die Größe der Region definiert - es kann sich um eine kleine Verwaltungseinheit oder um ein staatliches Territorium handeln -, womit dieses Paradigma eine geeignete Klammer zwischen Raumwirtschaftstheorie und Außenwirtschaftstheorie darzustellen scheint. Die Neue Ökonomische Geographie leitet ihre Resultate aus einem mikroökonomischen Totalmodell vom Dixit-Stiglitz-Typ [1977] mit heterogenen Gütern ab. Das erste räumliche Modell von Krugman [1991] verfügt neben spezifischen Produktionsfunktionen - economies of scale - über spezielle zweistufige Nutzenfunktionen. Die Resultate hinsichtlich der räumlichen Verteilung der wirtschaftlichen Aktivitäten können allerdings im ursprünglichen Modell nur numerisch abgeleitet werden. Die Richtung der Standortverlagerungen hängt unter sonst gleichen Bedingungen von den Ausprägungen der Transportkosten ab. Im Laufe der weiteren Forschung wurde es 
möglich, durch die Verwendung alternativer Nutzenfunktionen analytisch lösbare Modelle zu entwickeln (vgl. Forslid/Ottaviano [2003], Pflüger, M. [2004]).

Die wichtigsten Annahmen, die freilich nicht für alle, wenn auch für viele Modelle der NÖG gelten, sollen hier kurz zusammengefaßt werden. Es wird eine Ökonomie mit zwei Sektoren, einem landwirtschaftlichen und einem industriellen Sektor, angenommen. Der landwirtschaftliche Sektor produziert ein homogenes Gut unter konstanten Skalenerträgen und verkauft dieses Gut auf einem homogenen Markt. Der industrielle Sektor stellt eine große Anzahl in physischer (oder psychischer) Hinsicht unterschiedlicher Güter her und verkauft diese unter den Marktbedingungen der monopolistischen Konkurrenz. Die Arbeiter des landwirtschaftlichen Sektors sind immobil, die des industriellen Sektors wandern in die Region, die die höchsten Reallöhne aufweist. Die Transportkosten zwischen zwei Regionen werden im Sinne Samuelsons formuliert, ein Teil der zu transportierenden Güter wird als Ressource für ihren Transport benötigt. Die Annahme dieser sogenannten Eisberg-Technologie des Transports erübrigt die Modellierung eines Transportsektors. Transportkosten entstehen nur für industrielle Güter, nicht aber für die landwirtschaftlichen Güter. Es besteht die Möglichkeit verschiedener Standorte, wobei jede Firma an nur einem Standort in einer der beiden Regionen angesiedelt sein kann. Alle Konsumenten verfügen über die gleichen Präferenzen, die ihren Niederschlag in einer Nutzenfunktion vom Cobb-Douglas-Typ finden; sie enthält als Argumente die industriellen und die landwirtschaftlichen Güter. Die gesamte Gütermenge der industriellen Güter wird als ein Kontinuum von unterschiedlichen Varianten verstanden, die durch eine CES-Unternutzenfunktion zusammengefaßt werden.

Einen Überblick, wenngleich auch keine vollständige Darstellung des Modells, gibt die Skizze nach Brakman/Garretsen/van Marrewijk [2001], die die Zahlungsströme zusammenfaßt. Wie dargelegt, werden zwei Sektoren - Landwirtschaft und Industrie - mit zwei Gruppen von Arbeitern - landwirtschaftliche und industrielle - angenommen und zwei Regionen unterstellt, wobei die Größe der Räume nicht näher definiert ist. Da die landwirtschaftlichen Güter zwischen den Regionen nicht handelbar sind und in der Erzeugerregion verbraucht werden, richtet sich nur die Nachfrage nach industriellen Gütern aus beiden Regionen auf die in beiden Regionen produzierten Industrieerzeugnisse. Da die landwirtschaftlichen Arbeiter zwischen den Regionen immobil sind und nur die industriellen Arbeiter zwischen den Regionen wandern können, folgen aus industriellen Ballungen auch große Ansiedlungsgebiete der Industriearbeiter, die einen großen Markt für industrielle Güter bilden. Die monetären Ströme, denen nicht dargestellte reale Ströme in umgekehrter Richtung entsprechen, sind in der nachfolgenden Abbildung 1 verdeutlicht. 
hier: Abb. 1: Darstellung der Zahlungsströme

Die in der Graphik dargestellten Ströme erzeugen Anpassungsprozesse an ein räumliches Gleichgewicht. Nehmen wir an, in einer Region - beispielsweise in Region 1 - sind viele Nachfrager angesiedelt. Der Nachfrage folgend verlagern die Industriefirmen ihre Standorte in Region 1, in der hohe Löhne gezahlt werden können, die weitere industrielle Arbeiter veranlassen, in diese Region zu wandern. Damit wächst der Markt in Region 1 weiter an; diesen Effekt bezeichnet man als Nachfragevorteil. Große regionale Märkte haben aber ferner auch den Vorteil, daß viele unterschiedliche Güter erzeugt und angeboten und nicht aus anderen Regionen eingeführt werden müssen. Diese industriellen Güter weisen in der Region niedrigere Preise auf, da für sie die Transportkosten entfallen. Das dadurch im Vergleich zu anderen Regionen entstehende niedrigere Preisniveau erhöht die Reallöhne, die zu weiteren Zuwanderungen von industriellen Arbeitskräften führen; diesen Effekt bezeichnet man als Kostenvorteil. Beide Effekte verstärken sich zirkulär und würden eine vollständige Entleerung der Region 2 nach sich ziehen, wenn nicht gegenläufige Effekte auftreten würden. Eine wachsende räumliche Konzentration von Firmen in Region 1 führt zu einem sich verstärkenden Wettbewerb auf dem Gütermarkt und zu sinkenden Löhnen, die wiederum den Zuzug von Arbeitskräften verringern. Diesen Effekt bezeichnet man als Wettbewerbseffekt. Die in das Modell exogen eingeführte Annahme der räumlichen Immobilität der landwirtschaftlichen Arbeiter - es können auch die Arbeiter anderer spezieller Industrien sein (z. B. Grundstoffindustrie) - verhindert die völlige Entleerung des Hinterlandes.

Obwohl das Basis-Modell sich auf zwei Regionen beschränkt, kann eine allgemeine analytische Lösung nicht angegeben werden. Die Lösung des Modells basiert auf acht nichtlinearen Gleichungen, die simultan zu lösen sind. Wie immer in solchen Fällen bietet sich die numerische Simulation als Ausweg an. Ziel der Überlegungen ist es, einen Zusammenhang zwischen den Reallohndifferenzen hinsichtlich Region 1 und 2, die sich aus dem Modell ergeben, und der Aufteilung der industriellen Produktion auf Region 1 und 2 herzustellen. Die Reallohndifferenzen, die mit $\omega_{1}-\omega_{2}$ angegeben werden, stellen den Anreiz zur interregionalen Wanderung der industriellen Arbeitskräfte dar; ist die Differenz positiv, so wandern die Arbeiter in Region 1, ist sie negativ, so wandern sie in Region 2. Für die interregionale Verteilung der Industrie gilt: Ist $\lambda=1$, so befindet sich die gesamte industrielle Produktion in Region 1, ist $\lambda=0$, so ist die gesamte industrielle Produktion in Region 2 angesiedelt. Als Simulationsmethode wird die sequentielle Iteration angewendet, wobei zunächst einige Koeffizienten numerisch festgelegt und die Transportkosten vorgege- 
ben werden müssen. Die Kurven in den entsprechenden Diagrammen werden punktweise iterativ ermittelt.

Gibt man alternativ die Transportkosten $F=2,1, F=1,5$ und $F=1,7$ vor, so erhält man die nachstehenden Abbildungen 2 bis 4. Im Falle von $F=2,1$ ist die Reallohndifferenz für $0 \leq \lambda<0,5$ positiv und für $0,5<\lambda \leq 1$ negativ (Abb. 2). Dieser Sachverhalt bedeutet, daß bei einer geringen Industrialisierung der Region 1 industrielle Arbeiter aus Region 2 aufgrund der positiven Reallohndifferenzen zuwandern werden. Bei einer hohen Industrialisierung der Region 1 werden, ausgelöst durch eine negative Reallohndifferenz, Arbeiter in Region 2 abwandern. Bei gleichen Reallöhnen in beiden Regionen bleiben Wanderungen aus; dieses Gleichgewicht ist bei einer symmetrischen Verteilung der industriellen Produktion auf beide Regionen $(\lambda=1 / 2)$ gegeben. Der vorgegebene Transportkostensatz läßt keine industrielle Agglomeration entstehen.

hier: Abb. 2: Gleichgewicht bei hohen Transportkosten

Nimmt man in einem zweiten Fall $F=1,5$ an (Abb. 3), so steigt die Reallohndifferenz mit der Konzentration der Industrie in Region 1. Das Gleichgewicht bei $\lambda=1 / 2$ ist instabil, da eine geringfügig größere Konzentration der Industrie in Region 1 sowohl zu höheren Nominallöhnen als auch zu einem sinkenden Preisindex durch die Einsparung von Transportkosten führt. Daher wandern alle Industriearbeiter der positiven Reallohndifferenz folgend in Region 1 ein. Diese Reallohndifferenz bleibt bestehen; es bilden sich eine Industrieagglomeration in Region 1 und ein landwirtschaftliches Hinterland in Region 2 heraus. Das Ergebnis kehrt sich um, wenn in der Umgebung des instabilen Gleichgewichts die Reallohndifferenz negativ ist; die Wanderungen der Industriearbeiter in die entgegengesetzte Richtung lassen Region 1 zum landwirtschaftlichen Hinterland und Region 2 zur Industrieagglomeration werden.

hier: Abb. 3: Gleichgewicht bei niedrigen Transportkosten

In Abbildung 4 wird ein Transportkostensatz von $F=1,7$ angenommen, der zu fünf Gleichgewichten führt. Die Werte $\lambda=0, \lambda=0,5$ und $\lambda=1$ erzeugen stabile Gleichgewichte, wobei im ersten Fall wiederum eine vollständige Konzentration der Industrie in Region 2, im dritten Fall in Region 1 und im zweiten Fall eine Gleichverteilung der Industrie auf beide Regionen erzeugt werden. Die Gleichgewichte $\lambda_{A}$ und $\lambda_{B}$ sind instabil: Wird die Reallohndifferenz in der Umgebung von $\lambda_{A}(\approx 0,17)$ negativ oder in der Umgebung von $\lambda_{B}(\approx 0,83)$ positiv, so folgt daraus eine vollständige Konzentration der Industrie in einer 
der beiden Regionen. Wird die Reallohndifferenz in der Umgebung von $\lambda_{A}$ positiv oder in der Umgebung von $\lambda_{B}$ negativ, so erhält man eine Gleichverteilung der Industrie auf beide Regionen. Zusammenfassend kann gesagt werden, daß sich mit steigenden Transportkosten die Industrie gleichmäßig auf die Regionen verteilt und geringe Transportkosten die Herausbildung von industriellen Agglomerationen hervorrufen, genauer gesagt, sie bewirken die Konzentration der gesamten Industrie in einer Region.

\section{hier: Abb. 4: Gleichgewicht bei mittleren Transportkosten}

Es zeigt sich unter den angenommenen Parameterwerten, daß für den Wertebereich $F>1,63$ die Bedingung für ein stabiles symmetrisches Gleichgewicht gegeben ist. Die untere Grenze des Wertebereichs $\tilde{F}$ wird als "sustain point " bezeichnet; für den Wertebereich $0<F<1,81$ ist die Bedingung einer dauerhaften Agglomeration gegeben. Die obere Grenze des Wertebereichs $\bar{F}$ wird als "break point" bezeichnet. Diese Grenzen können in einem $\lambda$-F-Diagramm für ein Kontinuum von Transportkosten verdeutlicht werden (vgl. Abb. 5), wobei in der graphischen Darstellung alle durchgezogenen Linien stabile Gleichgewichte miteinander verbinden und alle gepunkteten Linien instabile Gleichgewichte. Die gesamten Wertebereiche betragen $\lambda \in[0,1]$ und $F \in[1, \infty)$. Ein stabiles räumliches Gleichgewicht erhält man für den Wertebereich der Transportkosten $0<F \leq \bar{F}$ und $\tilde{F} \leq F<\infty,(\tilde{F}<\bar{F})$, wobei im ersten Bereich die gesamte Industrie entweder in Region 1 oder $2(\lambda=1$ oder $\lambda=0)$ angesiedelt ist und im zweiten Bereich eine Gleichverteilung auf beide Regionen stabil ist. Soweit die Darstellung des Standardmodells der Neuen Ökonomischen Geographie.

hier: Abb. 5: Transportkosten und regionale Verteilung der Industrien

Betrachtet man das vorgestellte Modell, so lassen sich folgende Eigenschaften und Sachverhalte, die über die Aussagen der Abbildungen hinausgehen, zusammenfassend feststellen: Die Industriearbeiter wandern zu jener Region hin, die positive Reallohndifferenzen aufweist, wobei die industriellen Standorte diese räumliche Bewegung simultan nachvollziehen. Die Reallöhne entstehen durch die Gewichtung der Nominallöhne mit dem regionalen Preisniveau der industriellen Erzeugnisse. Die regionalen Preisniveaus enthalten ihrerseits neben den Nominallöhnen beider Regionen, die auf die Güterpreise zurückgeführt werden können, ferner die Transportkosten, mit denen die importierten Güter belastet sind. Ein hoher Anteil importierter Güter bedeutet ein hohes Preisniveau und niedrige Reallöhne. Ein hoher Anteil importierter Waren geht aber auch einher mit einer geringen industriellen 
Produktion in der Importregion und mit einer geringen Realisierung steigender Skalenerträge, was wiederum zu hohen Güterpreisen, zu einem hohen Preisniveau und niedrigen Reallöhnen führt. Eine Agglomeration aller industriellen Standorte in einer Region findet bei niedrigen Transportkosten statt, während hohe Transportkosten eine Gleichverteilung der Standorte auf beide Regionen erzeugen.

Die Agglomeration aller industriellen Standorte in einer Region und die industrielle Entleerung der anderen Region finden - wie man aus Abbildung 3 erkennen kann - bei niedrigen Transportkosten statt, die im Beispiel mit $F=1,5$ angenommen werden. Im Sinne des Eisberg-Theorems bedeutet das, daß 2/3 der Waren in der Zielregion ankommen oder, anders gesagt, die Ressourcen, die für den Transport aufgewendet werden müssen, betragen $50 \%$ der zu transportierenden Güter. (Im Fall von $F=2,1$ sind es $110 \%$.) In der Realität dürften aber kaum Transportkosten auftreten - auch nicht bei Baumaterial, übergroßen Spezialmaschinen oder Stahlkonstruktionen -, die $50 \%$ des Ab-Werk-Preises übersteigen. (Man kann einwenden, daß die Simulationsergebnisse auch von anderen numerischen Werten abhängen; jedoch sind diese nicht extrem unwahrscheinlich gewählt.) Ist $F$ sehr nahe 1, streben also die Transportkosten gegen Null, so wirken lediglich die steigenden Skalenerträge auf eine Industrieagglomeration hin.

Das in Kürze dargestellte Grundmodell der Neuen Ökonomischen Geographie, das von Krugman (1991) in die Diskussion eingeführt wurde und als "core-periphery-model" bezeichnet wird, ist in der Folgezeit in vielfacher Hinsicht verbessert und erweitert worden. Aus dieser Tatsache folgt, daß Einwände gegen das Grundmodell entweder durch die spätere theoretische Entwicklung entkräftigt werden können oder aber weiterhin bestehen. Zunächst soll aber hervorgehoben werden, worin der grundsätzliche wissenschaftliche Fortschritt des Ansatzes besteht: (1) Sowohl die Agglomeration (core) als auch die Entleerung des Hinterlandes (periphery) werden aus einem totalanalytischen Modell endogen erklärt. Damit wird die Erklärung wirtschaftlicher Zusammenballungen aus exogen eingeführten positiven externen Effekten abgelöst. Weitere Besonderheiten gegenüber der traditionellen Raumwirtschaftstheorie sind zu verzeichnen: (2) Die Heterogenität der Güter wird nicht nur in ihrer unterschiedlichen räumlichen Verfügbarkeit gesehen, sondern darüber hinaus auch in ihrer physischen Beschaffenheit oder psychischen Wahrnehmung. (3) Diese heterogenen Güter werden ausdrücklich unter wachsenden Skalenerträgen produziert, während in der überwiegenden Zahl der traditionellen raumwirtschaftlichen Modelle explizit oder implizit von konstanten Skalenerträgen ausgegangen wird.

Die theoretische Bewertung des Standardmodells der Neuen Ökonomischen Geogra- 
phie kann in zwei Stufen erfolgen. Zunächst ist das raumlose Dixit-Stiglitz-Modell zu betrachten und dann die raumwirtschaftliche Variante der NÖG. Die Vorzüge des DixitStiglitz-Modells sind zweifellos in der eindrucksvollen Verbindung der Märkte für Industriegüter, auf denen monopolistische Konkurrenz herrscht, mit einem mikroökonomischen Totalmodell bzw. einem allgemeinen Gleichgewichtsmodell zu sehen. Daß dabei alle Firmen eines Sektors und alle Haushalte als identisch angenommen werden, ist eine sinnvolle und übliche Vereinfachung, wobei, wie in jedem Modell der monopolistischen Konkurrenz mit repräsentativen, d. h. gleichartigen Firmen, die Heterogenität der hergestellten Güter keine Kostenunterschiede zur Folge haben dürfen (Beispiel: Eine Firma stellt rote Regenschirme her, die andere grüne, wobei die Farbunterschiede kostenneutral sind). Auf die fehlende Modellierung der einzelnen Firmen und die fehlende Möglichkeit ihres strategischen Verhaltens weist Neary [2001] nachdrücklich hin. Die Nutzenfunktion vom Cobb-DouglasTyp mit einem Element für Industriegüter, das einer CES-Funktion folgt, ist sehr speziell, aber, wie in der Literatur (Ottaviano/Tabuchi/Thisse [2002]) gezeigt wird, sind auch andere Funktionstypen möglich und mit dem Modell vereinbar. Als eine Einschränkung des Grundmodells muß zunächst die isoelastische Nachfragefunktion bezüglich einzelner industrieller Gütervarianten angesehen werden, die eine konstante Preiselastizität der Nachfrage aufweist. In späteren Ansätzen gelangt man allerdings durch veränderte Nutzenfunktionen zu linearen Nachfragefunktionen, die diese Eigenschaft bekanntlich nicht aufweisen.

In der raumwirtschaftlichen Version des Modells sind die Transportkosten, um die Berücksichtigung eines Transportsektors zu vermeiden, nach dem beschriebenen EisbergTheorem formuliert. Damit verzichtet man sowohl auf die fixen, mengenunabhängigen Transportkosten als auch auf die geographische Entfernung in den Modellen. Mehr noch, die Regionen, die klein sein können, aber auch Teile eines Landes oder sogar einzelne Volkswirtschaften umfassen können, haben keine räumliche Dimension. Innerhalb einer Region entstehen folglich keine Transportkosten, ungeachtet der Tatsache, daß diese höher sein können als die, die zwischen den Regionen anfallen. Damit folgt der Ansatz der traditionellen Außenwirtschaftstheorie, die - wenn überhaupt - Transportkosten nur zwischen zwei geographischen Punkten berücksichtigt. Innerhalb der industriellen Agglomeration können die Transportkosten durch die Überauslastung der Infrastruktur steigen und innerhalb des Hinterlandes auf Grund einer fehlenden leistungsfähigen Infrastruktur hoch sein. Im Standardmodell verursacht die nur innerhalb der Region anbietende Landwirtschaft ebenfalls keine Transportkosten. Diese Annahme ist bei der Art der Güter ebenso unwahrscheinlich wie die Unterstellung einer landwirtschaftlichen Produktion mit konstanten Skalenerträgen. Ferner ist das Preisniveau innerhalb einer Region einheitlich hoch, eine Folge der 
fehlenden Transportkosten für landwirtschaftliche und/oder industrielle Güterströme innerhalb einer Region. Schon Lösch [1938, 1939] hat in seiner Kritik an der Ricardianischen Außenwirtschaftstheorie auf die Uneinheitlichkeit intraregionaler Preisindizes hingewiesen und von der Möglichkeit sich räumlich, innerhalb der Länder fortpflanzender Preiswellen gesprochen. Schließlich sind die Gleichgewichte $\lambda=0,1$ und/oder $1 / 2 \mathrm{im}$ Standardmodell Alles-oder-Nichts-Lösungen, die vollständige Entleerung in einer und Industrieagglomerationen in einer anderen Region oder die völlige Gleichverteilung der Standorte auf beide Regionen erzeugen. Es fehlt ein gegenläufiger Modellmechanismus (Staukosten etwa), der eine - auch in der Realität zu beobachtende - Mehr-oder-Weniger-Lösung entstehen läßt. Dieser Mangel wurde ebenfalls in späteren Varianten durch verschiedene Modelländerungen beseitigt, so daß unsymmetrische, stabile Verteilungen der Industrien möglich sind (vgl. Fujita, M./Thisse, J.F. [2002]).

\section{Fazit}

Nunmehr soll die im Titel gestellte Frage beantwortet werden. Wenn man zusammenfaßt, gelangt man zu folgenden Einschätzungen. Die NÖG hat gegenüber der traditionellen Außenwirtschaftstheorie zum einen den Vorzug, die Transportkosten - die zu allgemeinen Transaktionskosten erweitert werden können - in die Modelle einzubeziehen. Damit wird ein theoretischer Mangel behoben. Zum anderen werden unterschiedliche Faktorausstattungen der Regionen bzw. Länder nicht wie in der traditionellen Außenhandelstheorie angenommen und die Handelsströme daraus abgeleitet, sondern es werden genau diese unterschiedlichen Faktorausstattungen durch die Höhe der Transportkosten erklärt. Es darf nicht übersehen werden, daß die Wohlfahrtswirkungen des internationalen Handels, ein Kernstück der traditionellen Handelstheorie, auch in fortgeschrittenen Modellen der NÖG mit Hilfe indirekter Nutzenfunktionen diskutiert werden können. Für die Raumwirtschaftstheorie besteht der Fortschritt zum einen in der modellendogenen Erklärung von Agglomerationen, die zum anderen aus einem Totalmodell bei heterogenen Gütern und economies of scale abgeleitet werden. Dieser Vorzug ist gleichzeitig auch Nachteil; die Bestimmung von Standorten einzelner Wirtschaftssubjekte, Industriefirmen, Haushalte, landwirtschaftlicher Betriebe, aber auch von Städten und öffentlichen Einrichtungen, ist mit den Instrumenten der NÖG nicht möglich. Der Versuch, Städtehierarchien abzuleiten, führt zu einer Modellkomplexität, die die Grenzen der NÖG aufzeigt (Fujita/Krugman/Venables [1999]). Ein Hauptmangel des neuen Ansatzes liegt in der Formulierung der Transportkosten selbst. Es ist nicht das Eisberg-Theorem gemeint, sondern die Tatsache, daß die Trans- 
portkosten einen gegebenen Betrag ausmachen, der nicht in geographische Entfernung und Transportkostensatz aufgespalten werden kann. Damit wird dem Modell die physischgeographische Grundlage entzogen, m.a.W., von den Transportkosten kann nicht mehr auf die geographische Entfernung zwischen den Wirtschaftsräumen geschlossen werden. Innerhalb der Wirtschaftsräume (Länder, Regionen, Subregionen) fallen keine Transportkosten an; diese können aber höher sein, als jene zwischen den Wirtschaftsräumen, und damit von ökonomischer Bedeutung. Würdigt man alle Gesichtspunkte, so muß man - so denke ich - zu folgendem Ergebnis kommen: Die Neue Ökonomische Geographie ergänzt in sehr wichtigen Punkten die traditionellen Theorien, Außenhandel und Raumwirtschaft, kann sie aber zur Zeit nicht ersetzen. Auch wenn neue Theorien, genauer gesagt ihre Vertreter, nicht selten zu einem Hegemonialanspruch bei der Erklärung von Wirklichkeit neigen, so kann doch eine genaue Analyse die tatsächliche Erklärungskraft ermitteln, die eindrucksvoll, aber nicht umfassend in dem Sinne ist, daß sie die traditionellen Raumwirtschaftsund Außenwirtschaftstheorie ablöst. Ein Paradigmenwechsel findet nicht statt. 


\section{Literatur}

Alonso, W. [1964], Location and Landuse, Cambridge Mass.

Brakman, S./Garretsen, H./van Marrewijk, C. [2001], An Introduction to Geographical Economics, Cambridge.

Christaller, W. [1933], Die zentralen Orte in Süddeutschland, Jena.

Dixit, A.K./Stiglitz, J.E. [1977], Monopolistic Competition and Optimum Product Diversity, American Economic Review, 67, 297-308.

Forslid, R./Ottaviano, I. P. [2003], An Analytically Solvable Core-Periphery Model, Journal of Economic Geography, 3, 229-240.

Fujita, M./Thisse, J.F. [2002], Economics of Agglomeration, Cambridge (Mass.), London.

Fujita, M./ Krugman, P./Venables A.J. [1999], The Spatial Economy: Cities, Regions and International Trade, Cambridge (Mass.), London.

Krugman, P.R. [1991], Geography and Trade, Cambridge (Mass.).

Kuhn, T. S. [1981], Die Struktur wissenschaftlicher Revolutionen, Frankfurt/Main.

Launhardt, W. [1885], Mathematische Begründung der Volkswirtschaftslehre, Leipzig.

Lösch, A. [1938], Wo gilt die Theorie der komparativen Kosten? Weltwirtschaftliches Arichiv, 48, 45-63.

Lösch, A. [1939], Eine neue Theorie des internationalen Handels, Weltwirtschaftliches Arichiv, 50, 308-326.

Lösch, A. [1944], Die räumliche Ordnung der Wirtschaft, 2. Aufl. Jena.

Neary, J.P. [2001], Of Hype and Hyperbolas: Introducing the New Economic Geography, Journal of Economic Literature, 39, 536-561. 
Ohlin, B. [1933], Interregional and International Trade, Cambridge (Mass.).

Ottaviano, G.I.P./Tabuchi, T./Thisse, J.F. [2002], Agglomeration and Trade revisited, International Economic Review, 43, 409-435.

Pflüger, M. [2004], A Simple, Analytically Solvable Chamberlinian Agglomeration Model, Regional Science and Urban Economics, 34, 565-573.

Schöler, K. [2005], Raumwirtschaftstheorie, München.

Steininger, K.W. [2001], International Trade and Transport, Cheltenham, Glos/Northampton, Mass.

von Thünen, J. H. [1930], Der isolierte Staat in Beziehung auf Landwirtschaft und Nationalökonomie, Neudruck Jena.

Weber A. [1909], Über den Standort der Industrie, Tübingen. 


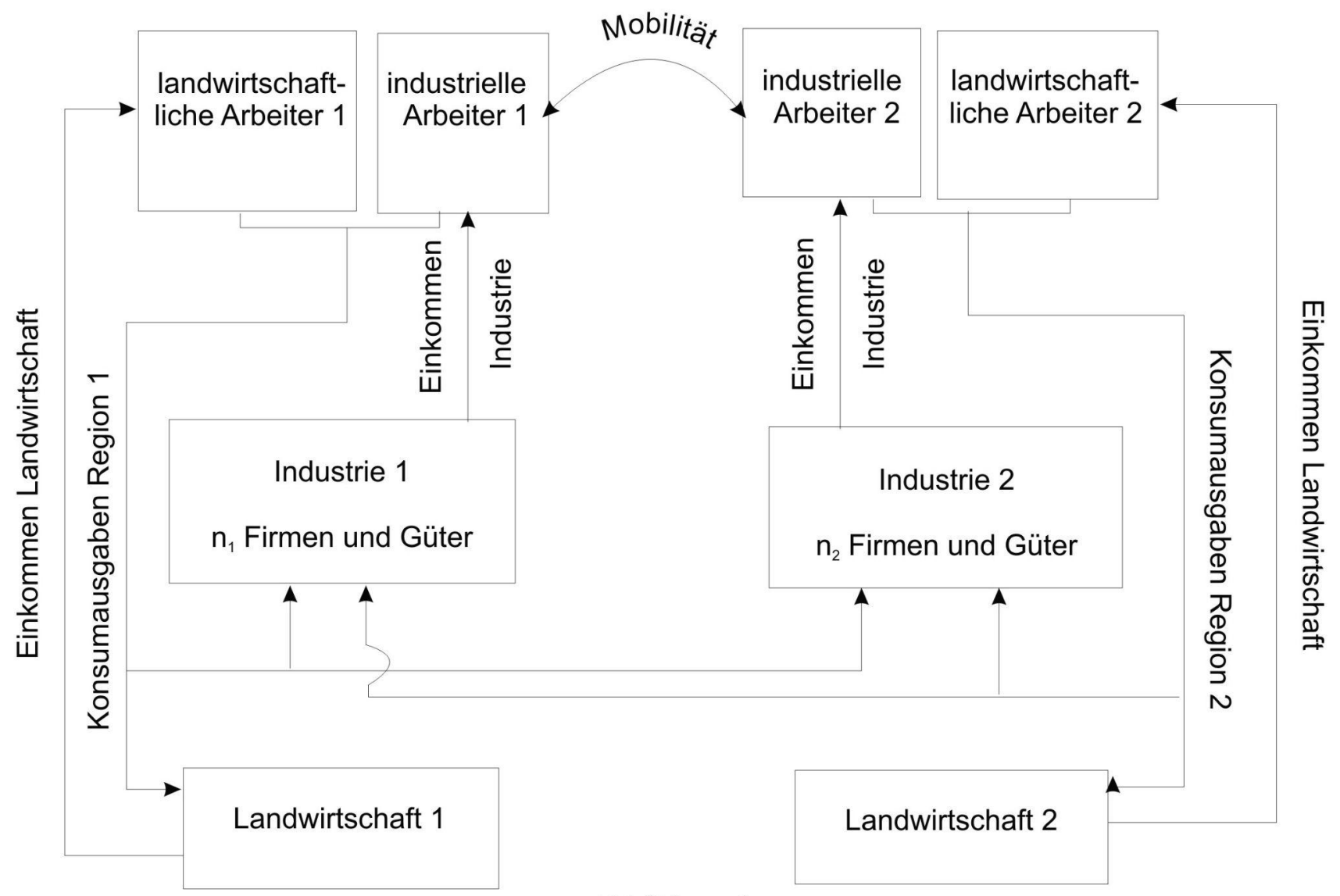

Abbildung 1

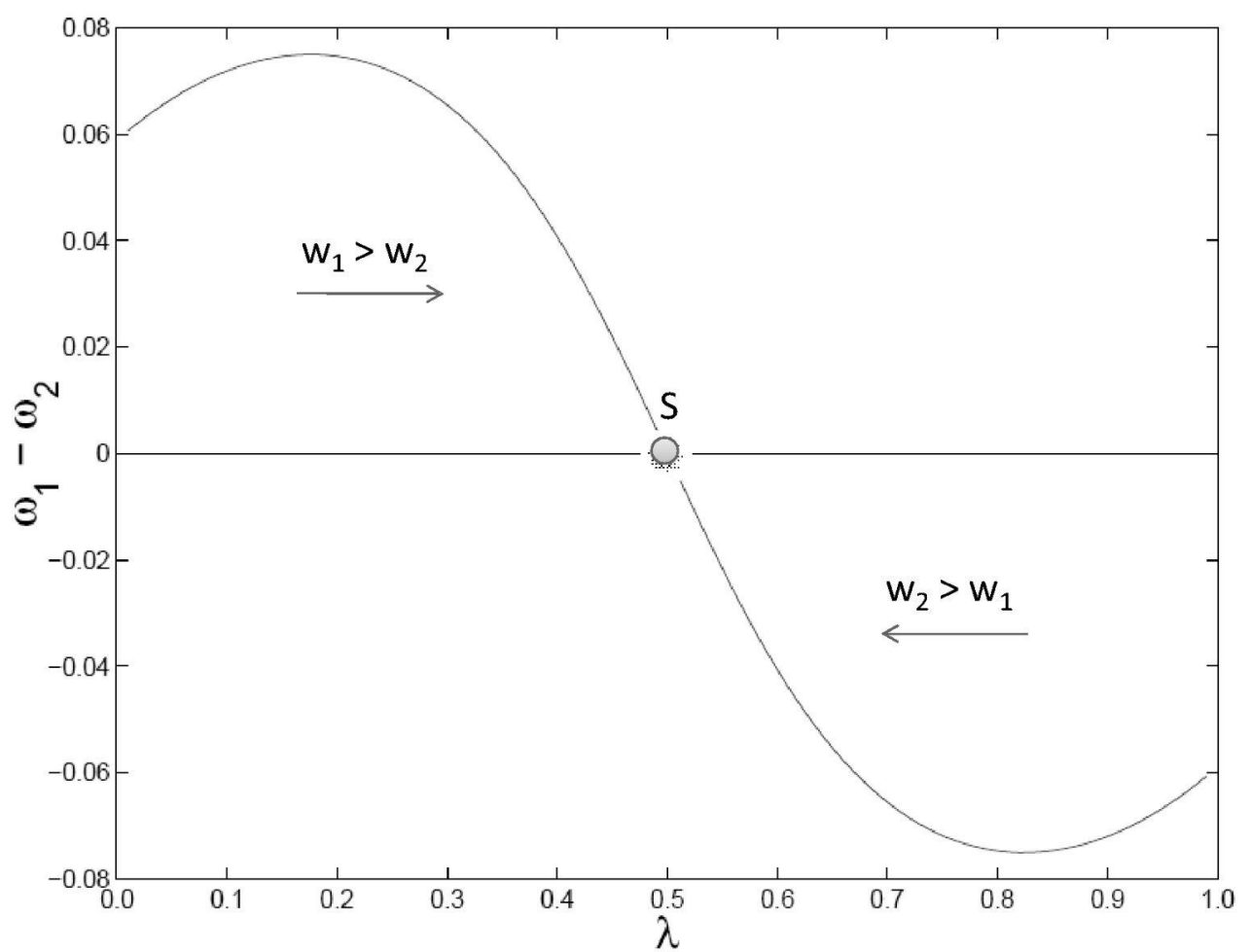

Abbildung 2 


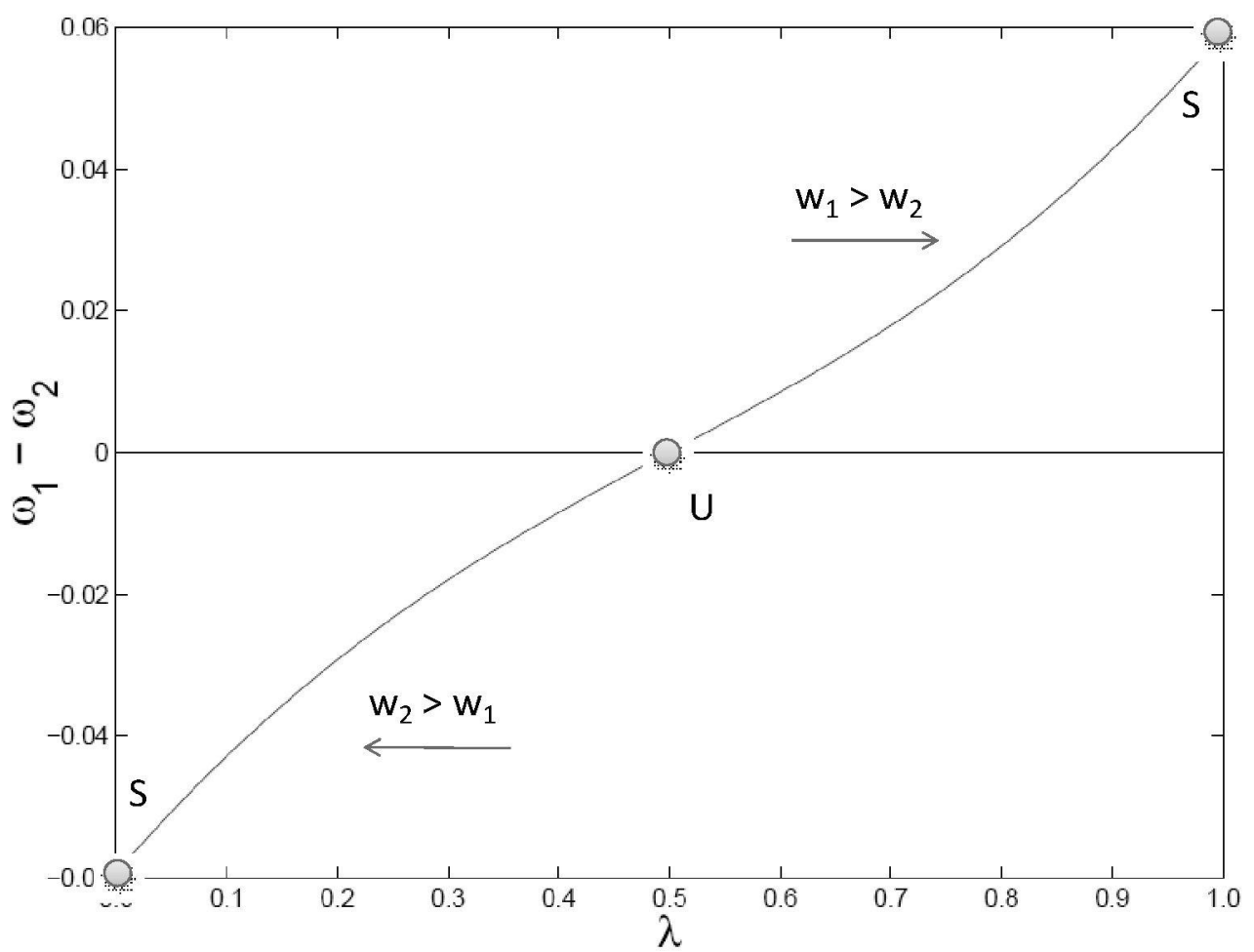

Abbildung 3

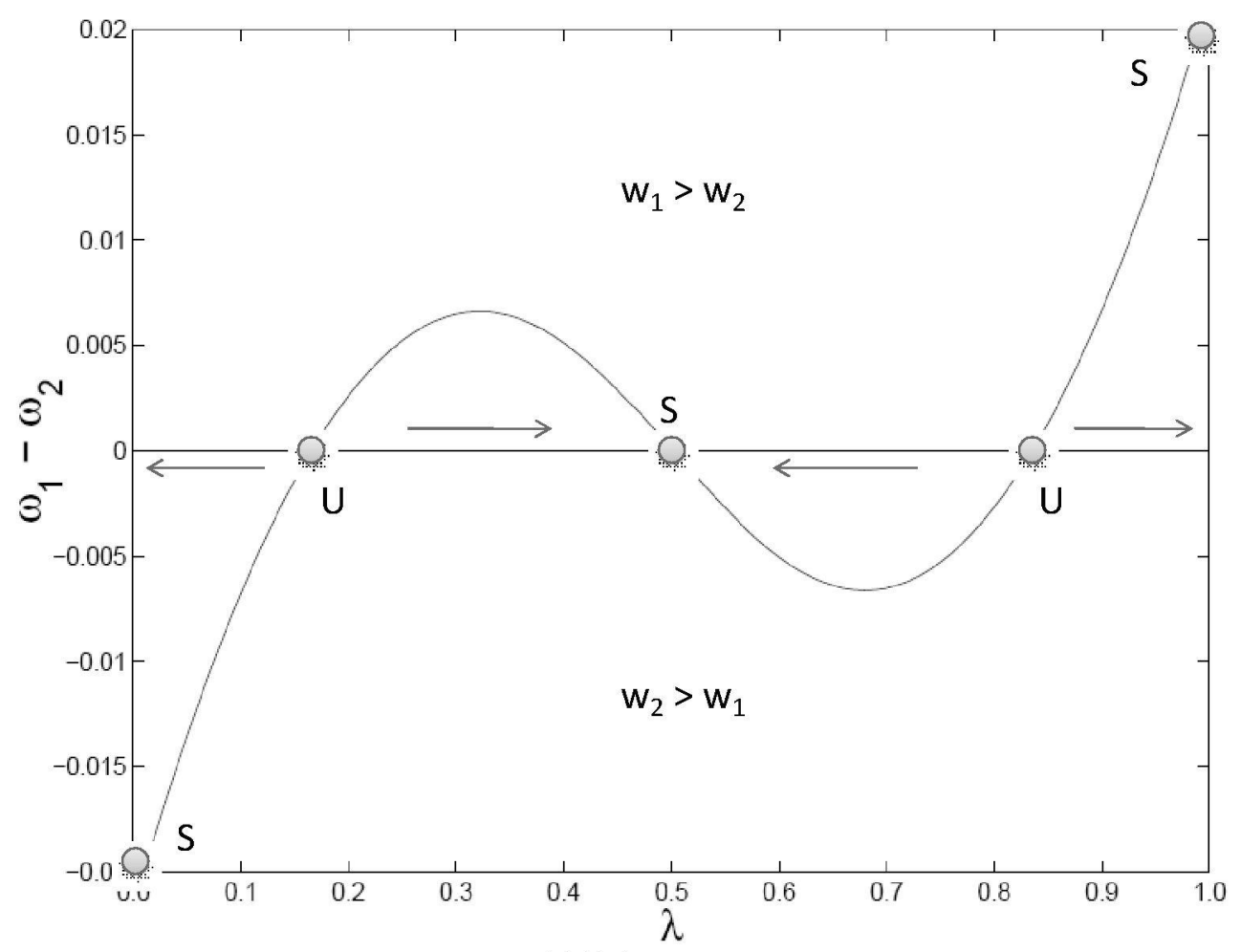

Abbildung 4 


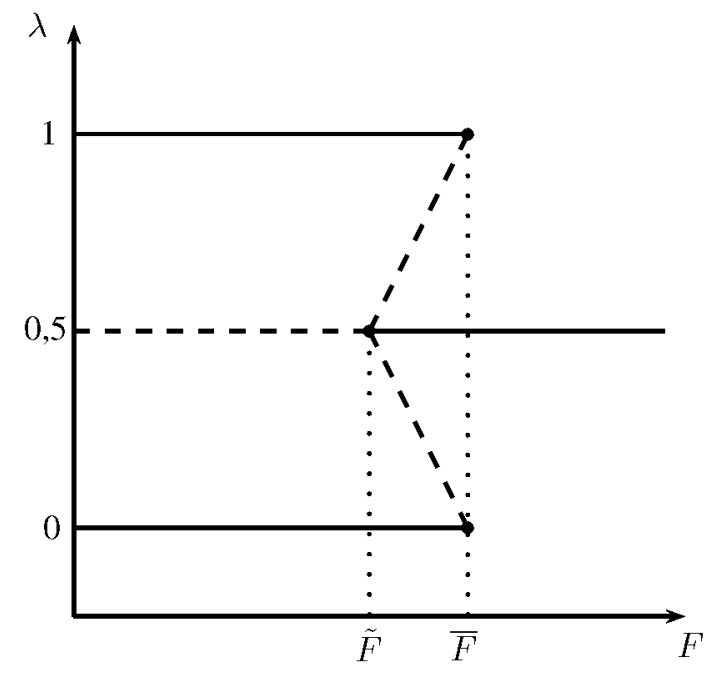

Abbildung 5 